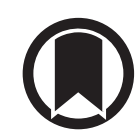

CrossMark

\title{
COVID-19 and biologics in severe asthma: data from the Belgian Severe Asthma Registry
}

\author{
To the Editor:
}

Epidemiological studies suggest that patients with asthma are not at an increased risk of severe coronavirus disease 2019 (COVID-19) caused by the novel coronavirus severe acute respiratory syndrome coronavirus 2 (SARS-CoV-2) [1-3]. Recent studies indicate that the severity of COVID-19 in patients with asthma is likely to depend on multiple factors. A type 2-low asthma phenotype, use of oral corticosteroids and severe asthma could be aggravating factors, while maintenance treatment with inhaled corticosteroids (ICS) and good asthma control are probably protective [4]. However, there is currently scarce information on the risk associated with COVID-19 in subjects with severe asthma and/or the use of biologics. Since eosinopenia is a biomarker for the severity of COVID-19 [5], the eosinophil depletion induced by anti-IL5 and anti-IL5 receptor blocking monoclonal antibodies raises concern in patients and their treating physicians. The handful of case reports about patients using the monoclonal antibodies omalizumab [6], benralizumab [7] or dupilumab for atopic dermatitis do offer a reassuring picture, but larger studies performed in patients with asthma are urgently needed. In immune-mediated inflammatory diseases, such as rheumatoid arthritis and psoriasis, treatment with biologics was not associated with worse COVID-19 outcomes [8]. Positioning papers and guidelines recommend asthma patients to maintain their regular treatments, including asthma biologics for those who qualify for them $[9,10]$, but epidemiological or clinical research data are scarce $[1,11]$.

The aim of our observational study was to evaluate the occurrence of COVID-19 in adult patients with severe asthma, based on data from the Belgian Severe Asthma Registry (BSAR), and to assess whether patients with severe asthma using biologics present an increased risk of severe COVID-19 as compared to those who do not use these medications.

The BSAR, a prospective cohort of patients with severe asthma defined according to the European Respiratory Society/American Thoracic Society criteria, has enrolled 1416 patients since 2009 [12, 13]. After obtaining the approval for an amendment from the BSAR's central ethics committee (UZ Gent; B67020084584), nine participating asthma centres contacted their patients. Of the 777 patients with severe asthma (68\% of patients with active follow-up in BSAR, i.e. with registry visits during the past 2 years) that were contacted, 676 (87\%) participated in this survey.

A standardised questionnaire was administered by phone or during outpatient visits and information about symptoms suggestive of COVID-19, hospital admission for COVID-19 and diagnostic testing through nasopharyngeal swab (PCR) or serology (SARS-CoV-2 IgG) was obtained. Patients were contacted between 30 April and 8 July 2020. Until 24 April, the government policy in Belgium was to limit PCR testing to healthcare workers or severe COVID-19 cases requiring hospitalisation. In case of milder symptoms, patients were put in quarantine but were not tested. From 24 April onwards, a greater proportion of samples were collected from symptomatic and asymptomatic people, as part of screening programmes, for instance prior to an intervention or hospitalisation. The monoclonal antibodies available for clinical use in severe asthma in Belgium are omalizumab, mepolizumab, benralizumab and reslizumab, while dupilumab is not yet reimbursed for asthma.

@ERSpublications

In a cohort of severe asthma patients, a small number of COVID-19 cases was found; none resulted in death or a very severe disease course. Use of biologics for severe allergic or severe eosinophilic asthma was not associated with a higher risk of COVID-19. https://bit.ly/3ndZmyD

Cite this article as: Hanon S, Brusselle G, Deschampheleire M, et al. COVID-19 and biologics in severe asthma: data from the Belgian Severe Asthma Registry. Eur Respir J 2020; 56: 2002857 [https://doi.org/ $10.1183 / 13993003.02857-2020]$. 
266 (39\%) of the 676 participants experienced at least one symptom suggestive of COVID-19 during the 4 months preceding the survey. Dyspnoea $(n=172 ; 25 \%)$, rhinorrhoea $(n=116 ; 17 \%)$, productive cough $(n=107 ; 16 \%)$, headache $(n=105 ; 16 \%)$ and chest pain $(n=69 ; 10 \%)$, were the most frequently reported symptoms. Only 43 patients (6\%) mentioned fever and only 35 (5\%) of the patients experienced sudden olfactory and gustative dysfunction, symptoms that are considered quite specific for COVID-19 [14], suggesting a low incidence of symptomatic COVID-19 in the study population.

Out of 676 patients, only 66 were tested by PCR on nasopharyngeal aspirates and nine (14\%) tested positive for SARS-CoV-2 (table 1). 40 (61\%) PCR tests were performed because the patients presented suggestive symptoms, whereas the remaining tests were done in the context of healthcare or contact screenings. All the patients that had a positive PCR test had presented with suggestive symptoms. As the national restrictions with regard to PCR testing might have led to an underestimation of the true incidence of SARS-CoV-2 infection in patients with severe asthma in Belgium, serology testing to determine SARS-CoV-2 IgG antibody levels was performed, at the discretion of the treating physician, in a subgroup of patients that had presented a combination of symptoms suggestive of COVID-19. Positive IgG serology was found in only eight out of 98 tested patients. Of note, three of these patients who showed positive SARS-CoV-2 IgG had also demonstrated a positive PCR test. Because serology testing for COVID-19 only became available for large-scale clinical use towards the end of our study period (June 2020), the number of cases identified by serology is probably also an underestimation of the true incidence. Overall, we identified 14 patients with COVID-19 infection confirmed by either PCR and/or specific IgG (table 1). Of these 14 patients, only five (all PCR-positive) had been hospitalised (with a short hospital stay ranging from 2 to 8 days). None presented with a severe asthma exacerbation or required treatment with systemic corticosteroids, admission to the intensive care unit, non-invasive ventilation, mechanical ventilation or extracorporeal membrane oxygenation, and there were no deaths. Only three patients received supplemental oxygen therapy (for 3 to 8 days). Chest computed tomography scan was performed in four hospitalised severe asthma patients and revealed pulmonary consolidations in three patients. Patients with confirmed COVID-19 reported a significantly higher median (interquartile range) number of symptoms than the remainder of the study population $(6(3-10)$ versus $2(1-3) ; \mathrm{p}<0.000001)$. Of the 35 patients that presented gustative or olfactory dysfunction, 24 were tested by means of PCR and/or serology and 10 (42\%) were found positive, a finding that is consistent with these symptoms being considered more specific for COVID-19 [14]. Importantly, there was no difference in incidence of COVID-19 between severe asthma patients treated with biologics (anti-IgE or anti-IL5/anti-IL5R) and patients not receiving any asthma biologic ( $>0.05)$ (table 1), but as the number of confirmed cases is small, this has to be interpreted cautiously. Recent use of oral corticosteroids (OCS) has been identified as a risk factor for

TABLE 1 Results of coronavirus disease 2019 (COVID-19) diagnostic testing and treatment of confirmed COVID-19 patients according to severe asthma treatment group

\begin{tabular}{|c|c|c|c|}
\hline & No biologic & Anti-lgE & Anti-IL5 or anti-IL5R \\
\hline All patients $\mathrm{n}$ & 242 & $\begin{array}{c}129 \\
\text { (omalizumab: } n=129 \text { ) }\end{array}$ & $\begin{array}{l}305 \\
\text { (benralizumab: } n=98, \\
\text { mepolizumab: } n=182 \text {, } \\
\text { reslizumab: } n=25 \text { ) }\end{array}$ \\
\hline \multicolumn{4}{|l|}{ PCR test for COVID-19 } \\
\hline Not done & 211 & 118 & 281 \\
\hline Positive & $3(1.2 \%)$ & 0 & $6(2 \%)$ \\
\hline Negative & 28 & 11 & 18 \\
\hline \multicolumn{4}{|l|}{ IgG test for COVID-19 } \\
\hline Not done & 241 & 99 & 238 \\
\hline Positive & 0 & $4(3.1 \%)$ & $4(1.3 \%)$ \\
\hline Negative & 1 & 26 & 63 \\
\hline Daily OCS n & $20(8.2 \%)$ & $10(7.8 \%)$ & $28(9.2 \%)$ \\
\hline Median dose mg (prednisone equivalent) & 4.5 & 5.0 & 5.0 \\
\hline Confirmed COVID-19 patients $n$ & 3 & 4 & 7 \\
\hline$\%$ of subgroup & $1.2^{\#}$ & $3.1^{\#}$ & $2.3^{\#}$ \\
\hline Hospitalisation $n$ & 1 & 0 & 4 \\
\hline Duration of hospitalisation days & 5 & NA & 4.5 (range 2-8) \\
\hline $\mathrm{O}_{2}$ therapy $\mathrm{n}$ & 1 & 0 & 2 \\
\hline Duration of $\mathrm{O}_{2}$ therapy days & 3 & NA & 5.5 (range 3-8) \\
\hline
\end{tabular}

NA: not applicable; OCS: oral corticosteroid. ${ }^{\#}$ : Chi-square test, $p>0.05$. 
COVID-19 related death [11], but in our cohort none of the 58 patients treated with maintenance OCS (median prednisone dose of $5 \mathrm{mg}$ ) were diagnosed with COVID-19.

To the best of our knowledge, this study is among the first to investigate the risk and severity of COVID-19 in a large cohort of well-characterised patients with severe asthma. The findings indicated a low incidence of COVID-19 infection in severe asthma patients (14 out of 676 patients; $2.1 \%$ ). Nevertheless, it is difficult to compare the incidence rate of confirmed COVID-19 cases in our BSAR cohort to the figure in the general population. Analysis of blood samples from Belgian blood donors revealed a seroprevalence reaching up to $5.1 \%$ [15]. Our study showed that only $2.1 \%$ of patients with severe asthma in Belgium had positive PCR or specific IgG to SARS-CoV-2 and that only 5\% presented COVID-19-related symptoms. Whether this relatively low incidence is due to those with severe asthma being more cautious than the general population and complying better with social distancing and other hygienic measures, or whether it is due to (patho) physiological features (type 2 inflammation) or beneficial effects of treatments such as ICS (the latter factors being associated with lower angiotensin-converting enzyme 2 expression) [16] remains to be elucidated.

Whereas viral respiratory tract infections are the most important cause of asthma exacerbations, none of the patients with confirmed COVID-19 in our BSAR cohort experienced an exacerbation. This is in line with the recent publication by BEURNIER et al. [1] reporting that, among hospitalised patients with severe pneumonia due to SARS-CoV-2 infection, patients with asthma were not overrepresented and did not present with an asthma exacerbation. The national restrictions with regard to PCR testing and the resulting possible underestimation of the true incidence of COVID-19 are a limitation of our study. Also, the participation in the survey of severe asthma patients already participating in a registry might have created some selection bias, limiting the generalisability of our data. On the other hand, the careful characterisation of the patients in our cohort is a strength of this research.

In conclusion, among this cohort of adult patients with severe asthma, a small number of COVID-19 cases was found, none of which resulted in death or a very severe disease course. Treatment with biologics for severe allergic or severe eosinophilic asthma was not associated with a higher risk of SARS-CoV-2 infection nor with more severe COVID-19. These data support the current practice to continue treatment with biologics in severe asthma during the COVID-19 pandemic.

Shane Hanon ${ }^{1}$, Guy Brusselle $\odot^{2}$, Maud Deschampheleire ${ }^{3}$, Renaud Louis ${ }^{4}$, Alain Michils ${ }^{5}$, Rudi Peché ${ }^{6}$, Charles Pilette, Peter Rummens ${ }^{8}$, Daniel Schuermans $\oplus^{1}$, Hélène Simonis ${ }^{3}$, Olivier Vandenplas ${ }^{9}$ and Florence Schleich ${ }^{4}$

${ }^{1}$ Respiratory Division, University Hospital UZ Brussel, Vrije Universiteit Brussel, Brussels, Belgium. ${ }^{2}$ Dept of Respiratory Medicine, Ghent University Hospital, Gent, Belgium. ${ }^{3}$ Dept of Respiratory Medicine, CHR Citadelle, Liege, Belgium. ${ }^{4}$ Dept of Respiratory Medicine, University Hospital of Liege, Liege, Belgium. ${ }^{5}$ Chest Dept, Erasme University Hospital, Université Libre de Bruxelles (ULB), Brussels, Belgium. ${ }^{6}$ Dept of Respiratory Medicine, ISPPC, CHU Charleroi, Charleroi, Belgium. ${ }^{7}$ Dept of Respiratory Medicine, Cliniques Universitaires St-Luc and institute of Experimental and Clinical Research, Université Catholique de Louvain (UCL), Brussels, Belgium. ${ }^{8}$ Dept of Respiratory Medicine, University Hospital Saint-Pierre, Université Libre de Bruxelles, Brussels, Belgium. ${ }^{9}$ Dept of Chest Medicine, Centre Hospitalier Universitaire UCL Namur, Cliniques Universitaires de Mont-Godinne, Université Catholique de Louvain, Yvoir, Belgium.

Correspondence: Shane Hanon, Respiratory Division, University Hospital UZ Brussel, Laarbeeklaan 101, 1090 Brussels, Belgium. E-mail: shane.hanon@uzbrussel.be

Received: 21 July 2020 | Accepted after revision: 27 Sept 2020

Author contributions: S. Hanon, G. Brusselle and F. Schleich conceived the study. D. Schuermans and all co-authors collected the data. S. Hanon and F. Schleich analysed the data, and co-wrote the manuscript. All authors provided critical feedback on the data, edited the manuscript and guarantee the accuracy of the data and the ensuing manuscript.

Conflict of interest: S. Hanon reports personal fees from GSK, AstraZeneca, Teva, Sanofi and Novartis, grants and personal fees from Chiesi, outside the submitted work. G. Brusselle reports personal fees from AstraZeneca, Boehringer Ingelheim, Chiesi, GSK, Novartis, Sanofi and Teva, outside the submitted work. M. Deschampheleire has nothing to disclose. R. Louis reports grants and personal fees from GSK, AstraZeneca and Novartis, grants from Chiesi, outside the submitted work. A. Michils reports grants, personal fees and non-financial support from AstraZeneca and Chiesi, grants and personal fees from GlaxoSmithKline, personal fees from Novartis, outside the submitted work. R. Peché has nothing to disclose. C. Pilette has nothing to disclose. P. Rummens has nothing to disclose. D. Schuermans has nothing to disclose. H. Simonis has nothing to disclose. O. Vandenplas has nothing to disclose. F. Schleich has nothing to disclose.

\section{References}

1 Beurnier A, Jutant EM, Jevnikar M, et al. Characteristics and outcomes of asthmatic patients with COVID-19 pneumonia who require hospitalisation. Eur Respir J 2020; 56: 2001875.

2 Docherty AB, Harrison EM, Green CA, et al. Features of 20133 UK patients in hospital with covid-19 using the ISARIC WHO Clinical Characterisation Protocol: prospective observational cohort study. BMJ 2020; 369: m1985. 
3 Chhiba KD, Patel GB, Vu THT, et al. Prevalence and characterization of asthma in hospitalized and non-hospitalized patients with COVID-19. J Allergy Clin Immunol 2020; 146: 307-314.

4 Maes T, Bracke K, Brusselle GG. Reply to: inhaled corticosteroids and COVID-19. Am J Respir Crit Care Med 2020; 202: 900-902.

5 Xia Z. Eosinopenia as an early diagnostic marker of COVID-19 at the time of the epidemic. EClinicalMedicine 2020; 23: 100398.

6 Lommatzsch M, Stoll P, Virchow JC. COVID-19 in a patient with severe asthma treated with omalizumab. Allergy 2020; 75: 2705-2708.

7 García-Moguel I, Díaz Campos R, Alonso Charterina S, et al. COVID-19, severe asthma, and biologics. Ann Allergy Asthma Immunol 2020; 125: 357-359.

8 Haberman R, Axelrad J, Chen A, et al. Covid-19 in immune-mediated inflammatory diseases - case series from New York. N Engl J Med 2020; 383: 85-88.

9 Morais-Almeida M, Aguiar R, Martin B, et al. COVID-19, asthma, and biologic therapies: what we need to know. World Allergy Organ J 2020; 13: 100126.

10 Vultaggio A, Agache I, Akdis CA, et al. Considerations on biologicals for patients with allergic disease in times of the COVID-19 pandemic: an EAACI statement. Allergy 2020; 75: 2764-2774.

11 Williamson EJ, Walker AJ, Bhaskaran K, et al. Factors associated with COVID-19-related death using OpenSAFELY. Nature 2020; 584: 430-436.

12 Schleich F, Brusselle G, Louis R, et al. Heterogeneity of phenotypes in severe asthmatics. The Belgian Severe Asthma Registry (BSAR). Respir Med 2014; 108: 1723-1732.

13 Chung KF, Wenzel SE, Brozek JL, et al. International ERS/ATS guidelines on definition, evaluation and treatment of severe asthma. Eur Respir J 2014; 43: 343-373.

14 Tong JY, Wong A, Zhu D, et al. The prevalence of olfactory and gustatory dysfunction in COVID-19 patients: a systematic review and meta-analysis. Otolaryngol Head Neck Surg 2020; 163: 3-11.

15 Sciensano. www.sciensano.be/nl. Date last accessed: 18 Jul 2020. Date last updated: 18 Jul 2020.

16 Peters MC, Sajuthi S, Deford P, et al. COVID-19-related genes in sputum cells in asthma. Relationship to demographic features and corticosteroids. Am J Respir Crit Care Med 2020; 202: 83-90. 